\title{
ON CONTACT GRAPHS OF TOTALLY SEPARABLE PACKINGS IN LOW DIMENSIONS
}

\author{
KÁROLY BEZDEK AND MÁRTON NASZÓDI
}

\begin{abstract}
The contact graph of a packing of translates of a convex body in Euclidean $d$-space $\mathbb{E}^{d}$ is the simple graph whose vertices are the members of the packing, and whose two vertices are connected by an edge if the two members touch each other. A packing of translates of a convex body is called totally separable, if any two members can be separated by a hyperplane in $\mathbb{E}^{d}$ disjoint from the interior of every packing element.

We give upper bounds on the maximum vertex degree (called separable Hadwiger number) and the maximum number of edges (called separable contact number) of the contact graph of a totally separable packing of $n$ translates of an arbitrary smooth convex body in $\mathbb{E}^{d}$ with $d=2,3,4$. In the proofs, linear algebraic and convexity methods are combined with volumetric and packing density estimates based on the underlying isoperimetric (resp., reverse isoperimetric) inequality.
\end{abstract}

\section{INTRODUCTION}

We denote the $d$-dimensional Euclidean space by $\mathbb{E}^{d}$, and the unit ball centered at the origin $\mathbf{o}$ by $\mathbf{B}^{d}$. A convex body $\mathbf{K}$ is a compact convex subset of $\mathbb{E}^{d}$ with nonempty interior. Throughout the paper, $\mathbf{K}$ always denotes a convex body in $\mathbb{E}^{d}$. If $\mathbf{K}=-\mathbf{K}:=\{-x: x \in \mathbf{K}\}$, then $\mathbf{K}$ is said to be $\mathbf{o}$-symmetric. $\mathbf{K}$ is said to be smooth if at every point on the boundary bd $\mathbf{K}$ of $\mathbf{K}$, the body $\mathbf{K}$ is supported by a unique hyperplane of $\mathbb{E}^{d}$. $\mathbf{K}$ is strictly convex if the boundary of $\mathbf{K}$ contains no nontrivial line segment.

The kissing number problem asks for the maximum number $k(d)$ of non-overlapping translates of $\mathbf{B}^{d}$ that can touch $\mathbf{B}^{d}$. Clearly, $k(2)=6$. To date, the only known kissing number values are $k(3)=12$ [20], $k(4)=24$ [16], $k(8)=240$ [17], and $k(24)=196560$ [17]. For a survey of kissing numbers we refer the interested reader to [7].

Generalizing the kissing number, the Hadwiger number or the translative kissing number $H(\mathbf{K})$ of a convex body $\mathbf{K}$ is the maximum number of non-overlapping translates of $\mathbf{K}$ that all touch K. Given the difficulty of the kissing number problem, determining Hadwiger numbers is highly nontrivial with few exact values known for $d \geq 3$. The best general upper and lower bounds on $H(\mathbf{K})$ are due to Hadwiger [12] and Talata [22] respectively, and can be expressed as

$$
2^{c d} \leq H(\mathbf{K}) \leq 3^{d}-1,
$$

where $c$ is an absolute constant and equality holds in the right inequality if and only if $\mathbf{K}$ is an affine $d$-dimensional cube [11].

A packing of translates of a convex domain, that is, a convex body $\mathbf{K}$ in $\mathbb{E}^{2}$ is said to be totally separable if any two packing elements can be separated by a line of $\mathbb{E}^{2}$ disjoint from

2010 Mathematics Subject Classification. (Primary) 05C10, 52C15, (Secondary) 05B40, 46B20.

Key words and phrases. Convex body, totally separable packing, Hadwiger number, separable Hadwiger number, contact graph, contact number, separable contact number. 
the interior of every packing element. This notion was introduced by G. Fejes Tóth and L. Fejes Tóth [10].

We can define a totally separable packing of translates of a $d$-dimensional convex body $\mathbf{K}$ in a similar way by requiring any two packing elements to be separated by a hyperplane in $\mathbb{E}^{d}$ disjoint from the interior of every packing element [6, 13].

Recall that the contact graph of a packing of translates of $\mathbf{K}$ is the simple graph whose vertices are the members of the packing, and whose two vertices are connected by an edge if and only if the two members touch each other. In this paper we investigate the maximum vertex degree (called separable Hadwiger number), as well as the maximum number of edges (called the maximum separable contact number) of the contact graphs of totally separable packings by a given number of translates of a smooth or strictly convex body $\mathbf{K}$ in $\mathbb{E}^{d}$. This extends and generalizes the results of [4] and [6]. The details follow.

1.1. Separable Hadwiger numbers. It is natural to introduce the totally separable analogue of the Hadwiger number as follows [4].

Definition 1.1. Let $\mathbf{K}$ be a convex body in $\mathbb{E}^{d}$. We call a family of translates of $\mathbf{K}$ that all touch $\mathbf{K}$ and, together with $\mathbf{K}$, form a totally separable packing in $\mathbb{E}^{d}$ a separable Hadwiger configuration of $\mathbf{K}$. The separable Hadwiger number $H_{\mathrm{sep}}(\mathbf{K})$ of $\mathbf{K}$ is the maximum size of a separable Hadwiger configuration of $\mathbf{K}$.

Recall that the Minkowski symmetrization of the convex body $\mathbf{K}$ in $\mathbb{E}^{d}$ denoted by $\mathbf{K}_{\mathbf{o}}$ is defined by $\mathbf{K}_{\mathbf{o}}:=\frac{1}{2}(\mathbf{K}+(-\mathbf{K}))=\frac{1}{2}(\mathbf{K}-\mathbf{K})=\frac{1}{2}\{\mathbf{x}-\mathbf{y}: \mathbf{x}, \mathbf{y} \in \mathbf{K}\}$. Clearly, $\mathbf{K}_{\mathbf{o}}$ is an o-symmetric $d$-dimensional convex body. Minkowski [15] showed that if $\mathcal{P}=\left\{\mathbf{x}_{1}+\mathbf{K}, \mathbf{x}_{2}+\right.$ $\left.\mathbf{K}, \ldots, \mathbf{x}_{n}+\mathbf{K}\right\}$ is a packing of translates of $\mathbf{K}$, then $\mathcal{P}_{\mathbf{o}}=\left\{\mathbf{x}_{1}+\mathbf{K}_{\mathbf{o}}, \mathbf{x}_{2}+\mathbf{K}_{\mathbf{o}}, \ldots, \mathbf{x}_{n}+\mathbf{K}_{\mathbf{o}}\right\}$ is a packing as well. Moreover, the contact graphs of $\mathcal{P}$ and $\mathcal{P}_{\mathbf{o}}$ are the same. Using the same method, it is easy to see that Minkowski's above statement applies to totally separable packings as well. (See also [4].) Thus, from this point on, we only consider o-symmetric convex bodies.

It is mentioned in [6] that based on [9] (see also, [18] and [14]) it follows in a straightforward way that $H_{\text {sep }}\left(\mathbf{B}^{d}\right)=2 d$ for all $d \geq 2$. On the other hand, if $\mathbf{K}$ is an o-symmetric convex body in $\mathbb{E}^{d}$, then each facet of the minimum volume circumscribed parallelotope of $\mathbf{K}$ touches $\mathbf{K}$ at the center of the facet and so, clearly $H_{\text {sep }}(\mathbf{K}) \geq 2 d$. Thus,

$$
2 d \leq H_{\text {sep }}(\mathbf{K}) \leq H(\mathbf{K}) \leq 3^{d}-1
$$

holds for any o-symmetric convex body $\mathbf{K}$ in $\mathbb{E}^{d}$. Furthermore, the $d$-cube is the only osymmetric convex body in $\mathbb{E}^{d}$ with separable Hadwiger number $3^{d}-1$ [11].

We investigate equality in the first inequality of (2). First, we note as an easy exercise that $H_{\text {sep }}$ as a map from the set of convex bodies equipped with any reasonable topology to the reals is upper semi-continuous. Thus, for any $d$, if an $\mathbf{o}$-symmetric convex body $\mathbf{K}$ in $\mathbb{E}^{d}$ is sufficiently close to the Euclidean ball $\mathbf{B}^{d}$ (say, $\mathbf{B}^{d} \subseteq \mathbf{K} \subseteq\left(1+\varepsilon_{d}\right) \mathbf{B}^{d}$, where $\varepsilon_{d}>0$ depends on $d$ only), then $H_{\text {sep }}(\mathbf{K})=2 d$.

Hence, it is natural to ask whether the set of those o-symmetric convex bodies in $\mathbb{R}^{d}$ with $H_{\text {sep }}(\mathbf{K})=2 d$ is dense. In this paper, we investigate whether $H_{\text {sep }}(\mathbf{K})=2 d$ holds for any o-symmetric smooth or strictly convex $\mathbf{K}$ in $\mathbb{E}^{d}$. Our first main result is a partial answer to this question. 
Definition 1.2. An Auerbach basis of an o-symmetric convex body $\mathbf{K}$ in $\mathbb{E}^{d}$ is a set of $d$ points on the boundary of $\mathbf{K}$ that form a basis of $\mathbb{E}^{d}$ with the property that the hyperplane through any one of them, parallel to the other $d-1$ supports $\mathbf{K}$.

Theorem 1. Let $\mathbf{K}$ be an $\mathbf{o - s y m m e t r i c ~ c o n v e x ~ b o d y ~ i n ~} \mathbb{E}^{d}$, which is smooth or strictly convex. Then

(a) For $d \in\{1,2,3,4\}$, we have $H_{\text {sep }}(\mathbf{K})=2 d$ and, in any separable Hadwiger configuration of $\mathbf{K}$ with $2 d$ translates of $\mathbf{K}$, the translation vectors are $d$ pairs of opposite vectors, where picking one from each pair yields an Auerbach basis of $\mathbf{K}$.

(b) $H_{\text {sep }}(\mathbf{K}) \leq 2^{d+1}-3$ for all $d \geq 5$.

We note that part (a) of Theorem 1 was proved for $d=2$ and smooth o-symmetric convex domains in [4]. We prove Theorem 1 in Section 3,

1.2. One-sided separable Hadwiger numbers. The one-sided Hadwiger number $h(\mathbf{K})$ of an $\mathbf{o}$-symmetric convex body $\mathbf{K}$ in $\mathbb{E}^{d}$ has been defined in [3] as the maximum number of non-overlapping translates of $\mathbf{K}$ that can touch $\mathbf{K}$ and lie in a closed supporting half-space of $\mathbf{K}$. It is proved in [3] that $h(\mathbf{K}) \leq 2 \cdot 3^{d-1}-1$ holds for any o-symmetric convex body $\mathbf{K}$ in $\mathbb{E}^{d}$ with equality for affine $d$-cubes only.

One could consider the obvious extension of the one-side Hadwiger number to separable Hadwiger configurations. However, a more restrictive and slightly more technical definition serves our purposes better, the reason of which will become clear in Theorem 2 and Example 3.1.

Definition 1.3. Let $\mathbf{K}$ be a smooth o-symmetric convex body in $\mathbb{E}^{d}$. The one-sided separable Hadwiger number $h_{\text {sep }}(\mathbf{K})$ of $\mathbf{K}$ is the maximum number $n$ of translates $2 \mathbf{x}_{1}+\mathbf{K}, \ldots, 2 \mathbf{x}_{n}+\mathbf{K}$ of $\mathbf{K}$ that form a separable Hadwiger configuration of $\mathbf{K}$, and the following holds. If $f_{1}, \ldots$, $f_{n}$ denote supporting linear functionals of $\mathbf{K}$ at the points $\mathbf{x}_{1}, \ldots, \mathbf{x}_{n}$, respectively, then $\mathbf{o} \notin \operatorname{conv}\left\{\mathbf{x}_{1}, \ldots, \mathbf{x}_{n}\right\}$ and $\mathbf{o} \notin \operatorname{conv}\left\{f_{1}, \ldots, f_{n}\right\}$.

Definition 1.4. For a positive integer $d$, let

$h_{\text {sep }}(d):=\max \left\{h_{\text {sep }}(\mathbf{K}): \mathbf{K}\right.$ is an o-symmetric, smooth and strictly convex body in $\left.\mathbb{E}^{d}\right\}$,

$H_{\text {sep }}(d):=\max \left\{H_{\text {sep }}(\mathbf{K}): \mathbf{K}\right.$ is an o-symmetric, smooth and strictly convex body in $\left.\mathbb{E}^{d}\right\}$, and set $H_{\text {sep }}(0)=h_{\text {sep }}(0)=0$.

The proof of part (国) of Theorem 1 relies on the following fact: for the smallest dimensional example $\mathbf{K}$ of an $\mathbf{o}$-symmetric, smooth and strictly convex body with $H_{\text {sep }}(\mathbf{K})>2 d$, we have $h_{\text {sep }}(\mathbf{K})>2 d$. More precisely,

\section{Theorem 2.}

(a) $h_{\mathrm{sep}}(d) \leq H_{\mathrm{sep}}(d) \leq \max \left\{2 \ell+h_{\mathrm{sep}}(d-\ell): \ell=0, \ldots, d\right\}$.

(b) $h_{\mathrm{sep}}(d)=d$ for $d \in\{1,2,3,4\}$.

(c) $h_{\mathrm{sep}}\left(\mathbf{B}^{d}\right)=d$ for the d-dimensional Euclidean ball $\mathbf{B}^{d}$ with $d \in \mathbb{Z}^{+}$.

According to Note 2.2, when bounding $H_{\text {sep }}(\mathbf{K})$ for a smooth or strictly convex body $\mathbf{K}$, it is sufficient to consider smooth and strictly convex bodies.

As a warning sign, in Example 3.1 we show that there is an o-symmetric, smooth and strictly convex body $\mathbf{K}$ in $\mathbb{E}^{5}$, which has a set of 6 translates that form a separable Hadwiger configuration, and the origin is not in the convex hull of the translation vectors.

We prove Theorem 2, and present Example 3.1 in Section 3 . 
1.3. Maximum separable contact numbers. Let $\mathbf{K}$ be an $\mathbf{o}$-symmetric convex body in $\mathbb{E}^{d}$, and let $\mathcal{P}:=\left\{\mathbf{x}_{1}+\mathbf{K}, \ldots, \mathbf{x}_{n}+\mathbf{K}\right\}$ be a packing of translates of $\mathbf{K}$. The number of edges in the contact graph of $\mathcal{P}$ is called the contact number of $\mathcal{P}$. Finally let $c(\mathbf{K}, n)$ denote the largest contact number of a packing of $n$ translates of $\mathbf{K}$ in $\mathbb{E}^{d}$. It is proved in [2] that $c(\mathbf{K}, n) \leq \frac{H(\mathbf{K})}{2} n-n^{\frac{d-1}{d}} g(\mathbf{K})$ holds for all $n>1$, where $g(\mathbf{K})>0$ depends on $\mathbf{K}$ only.

Definition 1.5. If $d, n \in \mathbb{Z}^{+}$and $\mathbf{K}$ is an o-symmetric convex body in $\mathbb{E}^{d}$, then let $c_{\text {sep }}(\mathbf{K}, n)$ denote the largest contact number of a totally separable packing of $n$ translates of $\mathbf{K}$.

According to Theorem 1, the maximum degree in the contact graph of a totally separable packing of a smooth convex body $\mathbf{K}$ is $2 d$, and hence, $c_{\text {sep }}(\mathbf{K}, n) \leq d n$, for $d \in\{1,2,3,4\}$. Our second main result is a stronger bound.

Theorem 3. Let $\mathbf{K}$ be a smooth $\mathbf{o - s y m m e t r i c ~ c o n v e x ~ b o d y ~ i n ~} \mathbb{E}^{d}$ with $d \in\{1,2,3,4\}$. Then

$$
c_{\text {sep }}(\mathbf{K}, n) \leq d n-n^{(d-1) / d} f(\mathbf{K})
$$

for all $n>1$, where $f(\mathbf{K})>0$ depends on $\mathbf{K}$ only.

In particular, if $\mathbf{K}$ is a smooth $\mathbf{o}$-symmetric convex domain in $\mathbb{E}^{2}$, then

$$
c_{\text {sep }}(\mathbf{K}, n) \leq 2 n-\frac{\sqrt{\pi}}{8} \sqrt{n}
$$

holds for all $n>1$.

In [4] it is proved that $c_{\text {sep }}(\mathbf{K}, n)=\lfloor 2 n-2 \sqrt{n}\rfloor$ holds for any o-symmetric smooth strictly convex domain $\mathbf{K}$ and any $n>1$. Thus, one may wonder whether the same statement holds for any smooth $\mathbf{o}$-symmetric convex domain $\mathbf{K}$.

We prove Theorem 3 in Section 4 . For a more explicit form of Theorem 3 see Theorem 4 in Section 4 .

1.4. Organization of the paper. In Section 2 we develop a dictionary that helps translate the study of separable Hadwiger configurations of smooth or strictly convex bodies to the language of systems of vector-linear functional pairs. In Section 3, based on our observations in Section 2, we prove Theorem 2, and show how our first main result, Theorem 1 follows from it.

In Section 4 we prove our second main result, Theorem 3. This proof is an adaptation of the proof of the main result of [2] to the setting of totally separable packings of smooth convex bodies. One of the main challenges of the adaptation is to compute the maximum vertex degree of the contact graph of a totally separable family of translates of a smooth convex body $\mathbf{K}$, and to characterize locally the geometric setting where this maximum is attained. This local characterization is provided by Theorem 1 .

Finally, in Section 5, we describe open problems and outline the difficulties in translating Theorem 3 to strictly convex (but, possibly not smooth) convex bodies.

\section{LinearizATiOn, FUndAMEnTAL PROPERTIES}

First, in order to give a linearization of the problem, we consider a set of $n$ pairs $\left(\mathbf{x}_{1}, f_{1}\right)$, $\ldots,\left(\mathbf{x}_{n}, f_{n}\right)$ where $\mathbf{x}_{i} \in \mathbb{E}^{d}$ and $f_{i}$ is a linear functional on $\mathbb{E}^{d}$ for all $1 \leq i \leq n$, and we define the following conditions that they may satisfy. 

$f_{i}\left(\mathbf{x}_{j}\right)=-1$, if and only if, $\mathbf{x}_{j}=-\mathbf{x}_{i}$ holds for all $1 \leq i, j \leq n, i \neq j$. $f_{i}\left(\mathbf{x}_{j}\right)=-1$, if and only if, $f_{j}=-f_{i}$ holds for all $1 \leq i, j \leq n, i \neq j$. $f_{i}\left(\mathbf{x}_{i}\right)=1$ and $f_{i}\left(\mathbf{x}_{j}\right) \in(-1,0]$ holds for all $1 \leq i, j \leq n, i \neq j$.

Lemma 2.1. There is an $\mathbf{o}$-symmetric, strictly convex body $\mathbf{K}$ in $\mathbb{E}^{d}$ with $H_{\text {sep }}(\mathbf{K}) \geq n$ if and only if, there is a set of $n$ vector-linear functional pairs $\left(\mathbf{x}_{1}, f_{1}\right), \ldots,\left(\mathbf{x}_{n}, f_{n}\right)$ in $\mathbb{E}^{d}$ satisfying (Lin) and (StrictC).

Similarly, there is an o-symmetric, smooth convex body $\mathbf{K}$ in $\mathbb{E}^{d}$ with $H_{\text {sep }}(\mathbf{K}) \geq n$ if and only if, there is a set of $n$ vector-linear functional pairs $\left(\mathbf{x}_{1}, f_{1}\right), \ldots,\left(\mathbf{x}_{n}, f_{n}\right)$ in $\mathbb{E}^{d}$ satisfying (Lin) and (Smooth).

Furthermore, the existence of an o-symmetric, smooth and strictly convex body with $H_{\mathrm{sep}}(\mathbf{K}) \geq n$ is equivalent to the existence of $n$ vector-linear functional pairs satisfying (Lin), (StrictCl) and (Smooth).

Proof of Lemma 2.1. Let $\mathbf{K}$ be an o-symmetric convex body in $\mathbb{E}^{d}$. Assume that $2 \mathbf{x}_{1}+$ $\mathbf{K}, 2 \mathbf{x}_{2}+\mathbf{K}, \ldots, 2 \mathbf{x}_{n}+\mathbf{K}$ is a separable Hadwiger configuration of $\mathbf{K}$, where $\mathbf{x}_{1}, \ldots, \mathbf{x}_{n} \in$ bd $\mathbf{K}$. For $1 \leq i \leq n$, let $f_{i}$ be the linear functional corresponding to the separating hyperplane of $\mathbf{K}$ and $2 \mathbf{x}_{i}+\mathbf{K}$ which is disjoint from the interior of all members of the family. That is, $f_{i}\left(\mathbf{x}_{i}\right)=1$ and $-1 \leq\left. f_{i}\right|_{K} \leq 1$.

Total separability yields that $f_{i}\left(\mathbf{x}_{j}\right) \in[-1,1] \backslash(0,1)$, for any $1 \leq i, j \leq n, i \neq j$. Suppose that $f_{i}\left(\mathbf{x}_{j}\right)=1$. Then $2 \mathbf{x}_{i}+\mathbf{K}$ and $2 \mathbf{x}_{j}+\mathbf{K}$ both touch the hyperplane $H:=\left\{x \in \mathbb{E}^{d}\right.$ : $\left.f_{i}(x)=1\right\}$ from one side, while $\mathbf{K}$ is on the other side of this hyperplane.

If $\mathbf{K}$ is strictly convex, then this is clearly not possible.

If $\mathbf{K}$ is smooth, then let $S$ be a separating hyperplane of $2 \mathbf{x}_{i}+\mathbf{K}$ and $2 \mathbf{x}_{j}+\mathbf{K}$ which is disjoint from int $\mathbf{K}$. Since $\mathbf{K}$ is smooth, $\mathbf{K} \cap H \cap S=\emptyset$, and hence, $\mathbf{K}$ does not touch $2 \mathbf{x}_{i}+\mathbf{K}$ or $2 \mathbf{x}_{j}+\mathbf{K}$, a contradiction.

Thus, if $\mathbf{K}$ is strictly convex or smooth, then (Lin) holds.

If $\mathbf{K}$ is strictly convex (resp., smooth), then (StrictC) (resp., (Smooth) ) follows immediately.

Next, assume that $\left(\mathbf{x}_{1}, f_{1}\right), \ldots,\left(\mathbf{x}_{n}, f_{n}\right)$ is a set of $n$ vector-linear functional pairs satisfying (Lin) and (StrictCl). We need to show that there is a strictly convex body $\mathbf{K}$ with $H_{\text {sep }}(\mathbf{K}) \geq$ $n$. Consider the $\mathbf{o}$-symmetric convex set $\mathbf{L}:=\left\{\mathbf{x} \in \mathbb{E}^{d}: f_{i}(\mathbf{x}) \in[-1,1]\right.$ for all $\left.1 \leq i \leq n\right\}$, the intersection of $n$ o-symmetric slabs.

Fix an $1 \leq i \leq n$. If there is no $j \neq i$ with $f_{j}\left(\mathbf{x}_{i}\right)=-1$, then $\mathbf{x}_{i}$ is in the relative interior of a facet of the polyhedral set $\mathbf{L}$, moreover, by (StrictC), no other point of the set $\left\{ \pm \mathbf{x}_{1}, \ldots, \pm \mathbf{x}_{n}\right\}$ lies on that facet.

If there is a $j \neq i$ with $f_{j}\left(\mathbf{x}_{i}\right)=-1$, then $\mathbf{x}_{i}$ is in the intersection of two facets of $\mathbf{L}$, moreover, by (StrictC), no other point of the set $\left\{ \pm \mathbf{x}_{1}, \ldots, \pm \mathbf{x}_{n}\right\}$ lies on the union of those two facets.

Thus, there is an $\mathbf{o}$-symmetric, strictly convex body $\mathbf{K} \subset \mathbf{L}$ which contains each $\mathbf{x}_{i}$. Clearly, for $1 \leq i \leq n$, the hyperplane $\left\{\mathbf{x} \in \mathbb{E}^{d}: f_{i}(\mathbf{x})=1\right\}$ supports $\mathbf{K}$ at $\mathbf{x}_{i}$. It is an easy exercise to see that the family $2 \mathbf{x}_{1}+\mathbf{K}, 2 \mathbf{x}_{2}+\mathbf{K}, \ldots, 2 \mathbf{x}_{n}+\mathbf{K}$ is a separable Hadwiger configuration of $\mathbf{K}$. 
Next, assume that $\left(\mathbf{x}_{1}, f_{1}\right), \ldots,\left(\mathbf{x}_{n}, f_{n}\right)$ is a set of $n$ vector-linear functional pairs satisfying (Lin) and (Smooth). To show that there is a smooth convex body $\mathbf{K}$ with $H_{\text {sep }}(\mathbf{K}) \geq n$, one may either copy the above proof and make the obvious modifications, or use duality: interchange the role of the $\mathbf{x}_{i} \mathrm{~s}$ with that of the $f_{i} \mathrm{~s}$, obtain a strictly convex body in the space of linear functionals, and then, by polarity obtain a smooth convex body in $\mathbb{E}^{d}$. We leave the details to the reader.

Finally, if (Lin), (StrictC) and (Smooth) hold, then in the above construction of a strictly convex body, we had that each point of the set $\left\{ \pm \mathbf{x}_{1}, \ldots, \pm \mathbf{x}_{n}\right\}$ lies in the interior of a facet of $\mathbf{L}$, with no other point lying on the same facet. Thus, there is an $\mathbf{o}$-symmetric, smooth and strictly convex body $\mathbf{K} \subset \mathbf{L}$ which contains each $\mathbf{x}_{i}$. Clearly, we have $H_{\text {sep }}(\mathbf{K}) \geq n$.

Note 2.2. Let $\mathbf{K}$ be an $\mathbf{o}$-symmetric, strictly convex body in $\mathbb{E}^{d}$, and consider a separable Hadwiger configuration of $\mathbf{K}$ with $n$ members. Then, by Lemma 2.1, we have a set of $n$ vector-linear functional pairs satisfying (Lin) and (StrictC).

If for each $1 \leq i \leq n$, we have that $-\mathbf{x}_{i}$ is not in the set of vectors, then OpenLin is automatically satisfied. We remark that in this case, we may replace $\mathbf{K}$ with a strictly convex and smooth body.

If for some $k \neq \ell$ we have $\mathbf{x}_{\ell}=-\mathbf{x}_{k}$, then by (Lin), $f_{j}\left(\mathbf{x}_{k}\right)=0$ for all $j \in[n] \backslash\{k, \ell\}$. Thus, if we remove $\left(\mathbf{x}_{k}, f_{k}\right)$ and $\left(\mathbf{x}_{\ell}, f_{\ell}\right)$ from the set of vector-linear functional pairs, then we obtain $n-2$ pairs that still satisfy (Lin) and (StrictC), and the linear functionals lie in a $(d-1)$-dimensional linear hyperplane. Thus, we may consider the problem of bounding their maximum number, $n-2$ in $\mathbb{E}^{d-1}$.

The same dimension reduction argument can be repeated when $\mathbf{K}$ is smooth. Thus, in order to bound $H_{\text {sep }}(\mathbf{K})$ for smooth or strictly convex bodies, it is sufficient to consider smooth and strictly convex bodies, and bound $n$ for which there are $n$ vectors with linear functionals satisfying OpenLin.

We will rely on the following basic fact from convexity due to Steinitz [21] in its original form, and then refined later with the characterization of the case of equality, see [19].

Lemma 2.3. Let $\mathbf{x}_{1}, \ldots, \mathbf{x}_{n}$ be points in $\mathbb{E}^{d}$ with $\mathbf{o} \in \operatorname{int} \operatorname{conv}\left\{\mathbf{x}_{1}, \ldots, \mathbf{x}_{n}\right\}$. Then there is a subset $A \subseteq\left\{\mathbf{x}_{1}, \ldots, \mathbf{x}_{n}\right\}$ of cardinality at most $2 d$ with $\mathbf{o} \in \operatorname{int} \operatorname{conv} A$.

Furthermore, if the minimal cardinality of such $A$ is $2 d$, then $A$ consists of the endpoints of d line segments which span $\mathbb{E}^{d}$, and whose relative interiors intersect in $\mathbf{o}$.

Proposition 2.4. Let $\left(\mathbf{x}_{1}, f_{1}\right), \ldots,\left(\mathbf{x}_{n}, f_{n}\right)$ be vector-linear functional pairs in $\mathbb{E}^{d}$ satisfying (Lin). Assume further that $\mathbf{o} \in \operatorname{int} \operatorname{conv}\left\{\mathbf{x}_{1}, \ldots, \mathbf{x}_{n}\right\}$. Then $n \leq 2 d$.

Moreover, if $n=2 d$, then the points $\mathbf{x}_{1}, \ldots, \mathbf{x}_{n}$ are vertices of a cross-polytope with center o.

Proof of Proposition 2.4. By (Lin), for any proper subset $A \subsetneq\left\{\mathbf{x}_{1}, \ldots, \mathbf{x}_{n}\right\}$, we have that the origin is not in the interior of conv $A$. Thus, by Lemma 2.3, $n \leq 2 d$.

Next, assume that $n=2 d$. Observe that it follows from (Lin) that if $x_{i}=\lambda x_{j}$ for some $1 \leq i, j \leq n, i \neq j$ and $\lambda \in \mathbb{R}$, then $\lambda=-1$. Thus, combining the argument in the previous paragraph with the second part of Lemma 2.3 yields the second part of Proposition 2.4.

Proposition 2.5. Let $\left(\mathbf{x}_{1}, f_{1}\right), \ldots,\left(\mathbf{x}_{n}, f_{n}\right)$ be vector-linear functional pairs in $\mathbb{E}^{d}$ satisfying OpenLin. Assume that $\mathbf{o} \notin \operatorname{conv}\left\{\mathbf{x}_{1}, \ldots, \mathbf{x}_{n}\right\}$. Then for any $1 \leq k<\ell \leq n$, the triangle $\operatorname{conv}\left\{\mathbf{o}, \mathbf{x}_{k}, \mathbf{x}_{\ell}\right\}$ is a face of the convex polytope $\mathbf{P}:=\operatorname{conv}\left(\left\{\mathbf{x}_{1}, \ldots, \mathbf{x}_{n}\right\} \cup\{\mathbf{o}\}\right)$. 
Proof of Proposition 2.5. By OpenLin, we have that $f_{i}\left(\mathbf{x}_{j}\right)>-1$ for all $1 \leq i, j \leq n, i \neq j$. Suppose for a contradiction that $\operatorname{conv}\left\{\mathbf{x}_{j}: j \in[n] \backslash\{k, \ell\}\right\}$ contains a point of the form $\mathbf{x}=\lambda \mathbf{x}_{k}+\mu \mathbf{x}_{\ell}$ with $\lambda, \mu \geq 0,0<\lambda+\mu \leq 1$. By (OpenLin), we have $f_{k}(\mathbf{x}), f_{\ell}(\mathbf{x}) \leq 0$. Thus,

$$
0 \geq f_{k}(\mathbf{x})+f_{\ell}(\mathbf{x})=\lambda\left(1+f_{\ell}\left(\mathbf{x}_{k}\right)\right)+\mu\left(1+f_{k}\left(\mathbf{x}_{\ell}\right)\right)>0,
$$

a contradiction.

\section{Proofs of Theorems 1 and 2}

Proof of Theorem (2. To prove part (国), we will use induction on $d$, the base case, $d=1$ being trivial. By the dimension-reduction argument in Note 2.2, we may assume that there are $n$ vector-linear functional pairs $\left(\mathbf{x}_{1}, f_{1}\right), \ldots,\left(\mathbf{x}_{n}, f_{n}\right)$ satisfying OpenLin).

If $\mathbf{o} \notin \operatorname{conv}\left\{\mathbf{x}_{1}, \ldots, \mathbf{x}_{n}\right\}$, and $\mathbf{o} \notin \operatorname{conv}\left\{f_{1}, \ldots, f_{n}\right\}$, then, clearly, $n \leq h_{\text {sep }}(d)$.

Thus, we may assume that $\mathbf{o} \in \operatorname{conv}\left\{\mathbf{x}_{1}, \ldots, \mathbf{x}_{n}\right\}$. We may also assume that $F=$ $\operatorname{conv}\left\{\mathbf{x}_{1}, \ldots, \mathbf{x}_{k}\right\}$ is the face of the polytope $\operatorname{conv}\left\{\mathbf{x}_{1}, \ldots, \mathbf{x}_{n}\right\}$ that supports $\mathbf{o}$, that is the face which contains $\mathbf{o}$ in its relative interior. Let $H:=\operatorname{span} F$. If $H$ is the entire space $\mathbb{E}^{d}$, then $\mathbf{o} \in \operatorname{int} \operatorname{conv}\left\{\mathbf{x}_{1}, \ldots, \mathbf{x}_{n}\right\}$ and hence, $n \leq 2 d$ follows from Proposition 2.4.

On the other hand, if $H$ is a proper linear subspace of $\mathbb{E}^{d}$, then clearly, for any $i>k$, we have that $f_{i}$ is identically zero on $H$.

Applying Proposition 2.4 on $H$ for $\left\{\mathbf{x}_{i}: i \leq k\right\}$ with $\left\{\left.f_{i}\right|_{H}: i \leq k\right\}$, we have

$$
k \leq 2 \operatorname{dim} H \text {. }
$$

Denote by $H^{\perp}$ the orthogonal complement of $H$, and by $P$ the orthogonal projection of $\mathbb{E}^{d}$ onto $H^{\perp}$. It is not hard to see that $P$ is one-to-one on the set $\left\{\mathbf{x}_{i}: i>k\right\}$. Moreover, the set of points $\left\{P \mathbf{x}_{i}: i>k\right\}$, with linear functionals $\left\{\left.f_{i}\right|_{H^{\perp}}: i>k\right\}$ restricted to $H^{\perp}$, satisfy (OpenLin in $H^{\perp}$.

Combining (3) with the induction hypothesis applied on $H^{\perp}$, we complete the proof of part (国).

For the three-dimensional bound in part (b), suppose that $\mathbf{o} \notin \operatorname{conv}\left\{\mathbf{x}_{1}, \ldots, \mathbf{x}_{4}\right\} \in \mathbb{E}^{3}$. By Radon's lemma, the set $\left\{\mathbf{o}, \mathbf{x}_{1}, \ldots, \mathbf{x}_{4}\right\}$ admits a partition into two parts whose convex hulls intersect contradicting Proposition [2.5. The same proof yields the two and the fourdimensional statements, while the one-dimensional claim is trivial.

We use a projection argument to prove part (ㄷ). Assume that $\mathbf{x}_{1}, \ldots, \mathbf{x}_{n}$ is a set of Euclidean unit vectors with $\left\langle\mathbf{x}_{i}, \mathbf{x}_{j}\right\rangle \in(-1,0]$ for all $1 \leq i, j \leq n, i \neq j$. Furthermore, let $\mathbf{y}$ be a unit vector with $\left\langle\mathbf{y}, \mathbf{x}_{i}\right\rangle>0$ for all $1 \leq i \leq n$. Consider the set of vectors $\mathbf{x}_{i}^{\prime}:=\mathbf{x}_{i}-\left\langle\mathbf{y}, \mathbf{x}_{i}\right\rangle \mathbf{y}, i=1, \ldots, n$, all lying in the hyperplane $\mathbf{y}^{\perp}$. Now, for $1 \leq i, j \leq n, i \neq j$, we have

$$
\left\langle\mathbf{x}_{i}^{\prime}, \mathbf{x}_{j}^{\prime}\right\rangle=\left\langle\mathbf{x}_{i}, \mathbf{x}_{j}\right\rangle-\left\langle\mathbf{y}, \mathbf{x}_{i}\right\rangle\left\langle\mathbf{y}, \mathbf{x}_{j}\right\rangle<0 .
$$

Thus, $\mathbf{x}_{i}^{\prime}, i=1, \ldots, n$ form a set of $n$ vectors in a $(d-1)$-dimensional space with pairwise obtuse angles. It is known [9, 18, 14], or may be proved using the same projection argument and induction on the dimension (projecting orthogonally to $\left(\mathbf{x}_{n}^{\prime}\right)^{\perp}$ ) that $n \leq d$ follows.

Example 3.1. By Lemma 2.1, it is sufficient to exhibit 6 vectors (with their convex hull not containing $\mathbf{o}$ in $\mathbb{E}^{5}$ ) and corresponding linear functionals satisfying (OpenLin). Let the unit vectors $\mathbf{v}_{4}, \mathbf{v}_{5}, \mathbf{v}_{6}$ be the vertices of an equilateral triangle centered at $\mathbf{o}$ in the linear plane $\operatorname{span}\left\{\mathbf{e}_{4}, \mathbf{e}_{5}\right\}$ of $\mathbb{E}^{5}$. Let $\mathbf{x}_{i}=\mathbf{e}_{i}$, for $i=1,2,3$, and let $\mathbf{x}_{i}=\left(\mathbf{e}_{1}+\mathbf{e}_{2}+\mathbf{e}_{3}\right) / 3+\mathbf{v}_{i}$, for $i=4,5,6$. Observe that $\mathbf{o} \notin \operatorname{conv}\left\{\mathbf{x}_{1}, \ldots, \mathbf{x}_{6}\right\}$, as $\left\langle\mathbf{e}_{1}+\mathbf{e}_{2}+\mathbf{e}_{3}, \mathbf{x}_{i}\right\rangle>0$ for $i=1, \ldots, 6$. 
We define the following linear functionals.

$f_{1}(\mathbf{x})=\left\langle\mathbf{e}_{1}-\frac{\mathbf{e}_{2}+\mathbf{e}_{3}}{2}, \mathbf{x}\right\rangle, f_{2}(\mathbf{x})=\left\langle\mathbf{e}_{2}-\frac{\mathbf{e}_{1}+\mathbf{e}_{3}}{2}, \mathbf{x}\right\rangle, f_{3}(\mathbf{x})=\left\langle\mathbf{e}_{3}-\frac{\mathbf{e}_{1}+\mathbf{e}_{2}}{2}, \mathbf{x}\right\rangle$, and $f_{i}(\mathbf{x})=$ $\left\langle\mathbf{v}_{i}, \mathbf{x}\right\rangle$, for $i=4,5,6$. Clearly, OpenLin holds.

Proof of Theorem 1. First, we prove part (国). If the origin is in the interior of the convex hull of the translation vectors, then Proposition 2.4 yields $n \leq 2 d$ and the characterization of equality. In the case when $\mathbf{o} \notin \operatorname{int} \operatorname{conv}\left\{\mathbf{x}_{i}\right\}$, Theorem 2 combined with Note 2.2 yields $n<2 d$.

The proof of part (b) follows closely a classical proof of Danzer and Grünbaum on the maximum size of an antipodal set in $\mathbb{E}^{d}[8]$.

By Lemma 2.1 and Note 2.2, we may assume that $\mathbf{K}$ is an $\mathbf{0}$-symmetric smooth strictly convex body in $\mathbb{E}^{d}$. Assume that $2 \mathbf{x}_{1}+\mathbf{K}, 2 \mathbf{x}_{2}+\mathbf{K}, \ldots, 2 \mathbf{x}_{n}+\mathbf{K}$ is a separable Hadwiger configuration of $\mathbf{K}$, where $\mathbf{x}_{1}, \ldots, \mathbf{x}_{n} \in \mathrm{bd} \mathbf{K}$. Let $f_{i}$ denote the linear functional corresponding to the hyperplane that separates $\mathbf{K}$ from $2 \mathbf{x}_{i}+\mathbf{K}$.

For each $1 \leq i \leq n$, let $\mathbf{K}_{i}$ be the set that we obtain by applying a homothety of ratio $1 / 2$ with center $\mathbf{x}_{i}$ on the set $\mathbf{K} \cap\left\{\mathbf{x} \in \mathbb{E}^{d}: f_{i}(\mathbf{x}) \geq 0\right\}$, that is,

$$
\mathbf{K}_{i}:=\frac{1}{2}\left(\mathbf{K} \cap\left\{\mathbf{x} \in \mathbb{E}^{d}: f_{i}(\mathbf{x}) \geq 0\right\}\right)+\frac{\mathbf{x}_{i}}{2} .
$$

These sets are pairwise non-overlapping. In fact, it is easy to see that the following even stronger statement holds:

$$
\left(\mu \mathbf{x}_{i}+\operatorname{int}\left(\frac{1}{2} \mathbf{K}\right)\right) \cap\left(\bigcup_{j \neq i} \mathbf{K}_{j}\right)=\emptyset
$$

for any $\mu \geq 0$ and $1 \leq i \leq n$. On the other hand, $\operatorname{vol}_{d}\left(\mathbf{K}_{i}\right)=2^{-(d+1)} \operatorname{vol}_{d}(\mathbf{K})$ by the central symmetry of $\mathbf{K}$, where $\operatorname{vol}_{d}(\cdot)$ stands for the $d$-dimensional volume of the given set. We remark that - unlike in the proof of the main result of [8] by Danzer and Grünbaum - the sets $\mathbf{K}_{i}$ are not translates of each other. Since each $\mathbf{K}_{i}$ is contained in $\mathbf{K} \backslash \operatorname{int}\left(\frac{1}{2} \mathbf{K}\right)$, we immediately obtain the bound $n \leq 2^{d+1}-2$.

To decrease the bound further, replace $\mathbf{K}_{1}$ by

$$
\widehat{\mathbf{K}}_{1}:=\mathbf{K} \cap\left\{\mathbf{x} \in \mathbb{E}^{d}: f_{1}(\mathbf{x}) \geq 1 / 2\right\} .
$$

Now, $\widehat{\mathbf{K}}_{1}, \mathbf{K}_{2}, \ldots, \mathbf{K}_{n}$ are still pairwise non-overlapping, and are contained in $\mathbf{K} \backslash$ int $\left(\frac{1}{2} \mathbf{K}\right)$. The smoothness of $\mathbf{K}$ yields $\widehat{\mathbf{K}}_{1} \supsetneq \mathbf{K}_{1}$, and hence, $\operatorname{vol}_{d}\left(\widehat{\mathbf{K}}_{1}\right)>2^{-(d+1)} \operatorname{vol}_{d}(\mathbf{K})$. This completes the proof of part (b) of Theorem 1 .

\section{Proof of Theorem 3}

We define a local version of a totally separable packing.

Definition 4.1. Let $\mathcal{P}:=\left\{\mathbf{x}_{i}+\mathbf{K}: i \in I\right\}$ be a finite or infinite packing of translates of $\mathbf{K}$, and $\rho>0$. We say that $\mathcal{P}$ is $\rho$-separable if for each $i \in I$ we have that the family $\left\{\mathbf{x}_{j}+\mathbf{K}: j \in I, \mathbf{x}_{j}+\mathbf{K} \subset \mathbf{x}_{i}+\rho \mathbf{K}\right\}$ is a totally separable packing of translates of $\mathbf{K}$. Let 
$\delta_{\text {sep }}(\rho, \mathbf{K})$ denote the largest density of a $\rho$-separable packing of translates of $\mathbf{K}$, that is,

$$
\delta_{\text {sep }}(\rho, \mathbf{K}):=\sup _{\mathcal{P}} \limsup _{\lambda \rightarrow \infty} \frac{\sum_{i: \mathbf{x}_{i}+\mathbf{K} \subset[-\lambda, \lambda]^{d}} \operatorname{vol}_{d}\left(\mathbf{x}_{i}+\mathbf{K}\right)}{(2 \lambda)^{d}},
$$

where the supremum is taken over all $\rho$-separable packings $\mathcal{P}$ of translates of $\mathbf{K}$.

We quote Lemma 1 of [5].

Lemma 4.2. Let $\left\{\mathbf{x}_{i}+\mathbf{K}: 1 \leq i \leq n\right\}$ be a $\rho$-separable packing of translates of an o-symmetric convex body $\mathbf{K}$ in $\mathbb{E}^{d}$ with $\rho \geq 1, n \geq 1$ and $d \geq 2$. Then

$$
\frac{n \operatorname{vol}_{d}(\mathbf{K})}{\operatorname{vol}_{d}\left(\bigcup_{1 \leq i \leq n} \mathbf{x}_{i}+2 \rho \mathbf{K}\right)} \leq \delta_{\text {sep }}(\rho, \mathbf{K}) .
$$

Lemma 4.3. Let $\mathbf{K}$ be a smooth $\mathbf{o - s y m m e t r i c ~ c o n v e x ~ b o d y ~ i n ~} \mathbb{E}^{d}$ with $d \in\{1,2,3,4\}$. Then there is a $\lambda>0$ such that for any separable Hadwiger configuration $\{\mathbf{K}\} \cup\left\{\mathbf{x}_{i}+\mathbf{K}: i=\right.$ $1, \ldots, 2 d\}$ of $\mathbf{K}$,

$$
\lambda \mathbf{K} \subseteq \bigcup_{i=1}^{2 d}\left(\mathbf{x}_{i}+\lambda \mathbf{K}\right) .
$$

holds. In particular, (4) holds with $\lambda=2$ when $d=2$.

Definition 4.4. We denote the smallest $\lambda$ satisfying (44) by $\lambda_{\text {sep }}(\mathbf{K})$, and note that $\lambda_{\text {sep }}(\mathbf{K})$ $\geq 2$, since otherwise $\bigcup_{i=1}^{2 d}\left(\mathbf{x}_{i}+\lambda \mathbf{K}\right)$ does not contain $\mathbf{o}$.

Proof of Lemma 4.3. Clearly, $\lambda$ satisfies (4) if and only if, for each boundary point $\mathbf{b} \in \operatorname{bd}(\mathbf{K})$ we have that at least one of the $2 d$ points $\mathbf{b}-\frac{2}{\lambda} \mathbf{x}_{i}$ is in $\mathbf{K}$.

First, we fix a separable Hadwiger configuration of $\mathbf{K}$ with $2 d$ members and show that for some $\lambda>0$, (44) holds. By Theorem [1, we have that $\left\{\mathbf{x}_{i}: i=1, \ldots, 2 d\right\}$ is an Auerbach basis of $\mathbf{K}$, and, in particular, the origin is in the interior of $\operatorname{conv}\left\{\mathbf{x}_{i}: i=1, \ldots, 2 d\right\}$. It follows from the smoothness of $\mathbf{K}$ that for each boundary point $\mathbf{b} \in \mathrm{bd}(\mathbf{K})$ we have that at least one of the $2 d$ rays $\left\{\mathbf{b}-t \mathbf{x}_{i}: t>0\right\}$ intersects the interior of $\mathbf{K}$. The existence of $\lambda$ now follows from the compactness of $\mathbf{K}$.

Next, since the set of Auerbach bases of $\mathbf{K}$ is compact (consider them as points in $\mathbf{K}^{d}$ ), it follows in a straightforward way that there is a $\lambda>0$, for which (4) holds for all separable Hadwiger configurations of $\mathbf{K}$ with $2 d$ members.

To prove the part concerning $d=2$, we make use of the characterization of the equality case in Part (a) of Theorem 1, An Auerbach basis of a planar o-symmetric convex body $\mathbf{K}$ means that $\mathbf{K}$ is contained in an $\mathbf{o}$-symmetric parallelogram, the midpoints of whose edges are $\pm \mathbf{x}_{1}, \pm \mathbf{x}_{2}$, and $\pm \mathbf{x}_{1}, \pm \mathbf{x}_{2} \in \mathbf{K}$. We leave it as an exercise to the reader that in this case, for each boundary point $\mathbf{b} \in \operatorname{bd}(\mathbf{K})$ we have that at least one of the 4 points $\mathbf{b} \pm \frac{\mathbf{x}_{1}}{2}, \mathbf{b} \pm \frac{\mathbf{x}_{2}}{2}$ is in $\mathbf{K}$.

We denote the $(d-1)$-dimensional Hausdorff measure by $\operatorname{vol}_{d-1}(\cdot)$, and the isoperimetric ratio of a bounded set $\mathbf{S} \subset \mathbb{E}^{d}$ for which it is defined as

$$
\operatorname{Iq}(\mathbf{S}):=\frac{\left(\operatorname{vol}_{d-1}(\mathrm{bd} \mathbf{S})\right)^{d}}{\underset{9}{\left(\operatorname{vol}_{d}(\mathbf{S})\right)^{d-1}}}
$$


and recall the isoperimetric inequality, according to which it is minimized by Euclidean balls, that is, $\operatorname{Iq}\left(\mathbf{B}^{d}\right) \leq \operatorname{Iq}(\mathbf{S})$ for any bounded set $\mathbf{S} \subset \mathbb{E}^{d}$, for which $\operatorname{Iq}(\mathbf{S})$ is defined.

Finally, we are ready to state our main result, from which Theorem 3 immediately follows.

Theorem 4. Let $\mathbf{K}$ be a smooth $\mathbf{o - s y m m e t r i c ~ c o n v e x ~ b o d y ~ i n ~} \mathbb{E}^{d}$ with $d \in\{1,2,3,4\}$. Then

$$
\begin{gathered}
c_{\mathrm{sep}}(\mathbf{K}, n) \leq \\
d n-\frac{n^{(d-1) / d}}{2\left[\lambda_{\mathrm{sep}}(\mathbf{K})\right]^{d-1}\left[\delta_{\mathrm{sep}}\left(\frac{\lambda_{\mathrm{sep}}(\mathbf{K})}{2}, \mathbf{K}\right)\right]^{(d-1) / d}}\left[\frac{\mathrm{Iq}\left(\mathbf{B}^{d}\right)}{\operatorname{Iq}(\mathbf{K})}\right]^{1 / d} \leq \\
d n-\frac{n^{(d-1) / d}\left(\operatorname{vol}_{d}\left(\mathbf{B}^{d}\right)\right)^{1 / d}}{4\left[\lambda_{\mathrm{sep}}(\mathbf{K})\right]^{d-1}}
\end{gathered}
$$

for all $n>1$.

In particular, in the plane, we have

$$
c_{\text {sep }}(\mathbf{K}, n) \leq 2 n-\frac{\sqrt{\pi}}{8} \sqrt{n}
$$

for all $n>1$.

Proof of Theorem 4. Let $\mathcal{P}=C+\mathbf{K}$ be a totally separable packing of translates of $\mathbf{K}$, where $C$ denotes the set of centers $C=\left\{\mathbf{x}_{1}, \ldots, \mathbf{x}_{n}\right\}$. Assume that $m$ of the $n$ translates is touched by the maximum number, that is, by Theorem $1, H_{\text {sep }}(\mathbf{K})=2 d$ others. By Lemma 4.3. we have

$$
\begin{gathered}
\operatorname{vol}_{d-1}\left(\operatorname{bd}\left(C+\lambda_{\text {sep }}(\mathbf{K}) \mathbf{K}\right)\right) \leq \\
(n-m)\left(\lambda_{\text {sep }}(\mathbf{K})\right)^{d-1} \operatorname{vol}_{d-1}(\operatorname{bd}(\mathbf{K})) .
\end{gathered}
$$

By the isoperimetric inequality, we have

$$
\operatorname{Iq}\left(\mathbf{B}^{d}\right) \leq \operatorname{Iq}\left(C+\lambda_{\text {sep }}(\mathbf{K}) \mathbf{K}\right)=\frac{\left(\operatorname{vol}_{d-1}\left(\mathrm{bd}\left(C+\lambda_{\text {sep }}(\mathbf{K}) \mathbf{K}\right)\right)\right)^{d}}{\left(\operatorname{vol}_{d}\left(C+\lambda_{\text {sep }}(\mathbf{K}) \mathbf{K}\right)\right)^{d-1}} .
$$

Combining (59) and (6) yields

$$
n-m \geq \frac{\left(\mathrm{Iq}\left(\mathbf{B}^{d}\right)\right)^{1 / d}\left[\operatorname{vol}_{d}\left(C+\lambda_{\text {sep }}(\mathbf{K}) \mathbf{K}\right)\right]^{(d-1) / d}}{\left(\lambda_{\text {sep }}(\mathbf{K})\right)^{d-1} \operatorname{vol}_{d-1}(\mathrm{bd} \mathbf{K})} .
$$

The latter, by Lemma 4.2 is at least

$$
\frac{\left(\mathrm{Iq}\left(\mathbf{B}^{d}\right)\right)^{1 / d}\left[\frac{n \operatorname{vol}_{d}(\mathbf{K})}{\delta_{\operatorname{sep}}\left(\lambda_{\mathrm{sep}}(\mathbf{K}) / 2, \mathbf{K}\right)}\right]^{(d-1) / d}}{\left(\lambda_{\text {sep }}(\mathbf{K})\right)^{d-1} \operatorname{vol}_{d-1}(\mathrm{bd} \mathbf{K})} .
$$

After rearrangement, we obtain the desired bound on $n$ completing the proof of the first inequality in Theorem 4 .

To prove the second inequality, we adopt the proof of [2, Corollary 1]. First, note that $\delta_{\text {sep }}\left(\frac{\lambda_{\operatorname{sep}}(\mathbf{K})}{2}, \mathbf{K}\right) \leq 1$, and $\left(\operatorname{Iq}\left(\mathbf{B}^{d}\right)\right)^{1 / d}=d \operatorname{vol}_{d}\left(\mathbf{B}^{d}\right)$. Next, according to Ball's reverse isoperimetric inequality [1, for any convex body $\mathbf{K}$, there is a non-degenerate affine map $T: \mathbb{E}^{d} \rightarrow \mathbb{E}^{d}$ with $\operatorname{Iq}(T \mathbf{K}) \leq(2 d)^{d}$. Finally, notice that $c_{\text {sep }}(\mathbf{K}, n)=c_{\text {sep }}(T \mathbf{K}, n)$, and the inequality follows in a straightforward way.

The planar bound follows by substituting the value $\lambda_{\text {sep }}(\mathbf{K})=2$ from Lemma 4.3 . 


\section{REMARKS}

Lemma 4.3 does not hold for strictly convex but not smooth convex bodies. Indeed, in $\mathbb{E}^{3}$, consider the $\mathbf{o}$-symmetric polytope $\mathbf{P}:=\operatorname{conv}\left\{ \pm \mathbf{e}_{1}, \pm \mathbf{e}_{2}, \pm \mathbf{e}_{3}, \pm 0.9\left(\mathbf{e}_{1}+\mathbf{e}_{2}+\mathbf{e}_{3}\right)\right\}$ where the $\mathbf{e}_{i} \mathrm{~s}$ are the standard basis vectors. The six translation vectors $\pm 2 \mathbf{e}_{1}, \pm 2 \mathbf{e}_{2}, \pm 2 \mathbf{e}_{3}$ generate a separable Hadwiger configuration of $\mathbf{P}$. For the vertex $\mathbf{b}:=0.9\left(\mathbf{e}_{1}+\mathbf{e}_{2}+\mathbf{e}_{3}\right)$, we have that each of the 3 lines $\left\{\mathbf{b}+t \mathbf{e}_{i}: t \in \mathbb{R}\right\}$ intersect $\mathbf{P}$ in $\mathbf{b}$ only. Thus, there is a strictly convex o-symmetric body $\mathbf{K}$ with the following properties. $\mathbf{P} \subset \mathbf{K}$, and $\pm \mathbf{e}_{i}$ is a boundary point of $\mathbf{K}$ for each $i=1,2,3$, and at $\pm \mathbf{e}_{i}$, the plane orthogonal to $\mathbf{e}_{i}$ is a support plane of $\mathbf{K}$, and $\mathbf{b}$ is a boundary point of $\mathbf{K}$, and the 3 lines $\left\{\mathbf{b}+t \mathbf{e}_{i}: t \in \mathbb{R}\right\}$ intersect $\mathbf{K}$ in $\mathbf{b}$ only. For this strictly convex $\mathbf{K}$, we have $\lambda_{\text {sep }}(\mathbf{K})=\infty$.

Thus, it is natural to ask if in Theorem 3 smoothness can be replaced by strict convexity. We note that in our proof, Lemma 4.3 is the only place which does not carry over to this case.

The same construction of the polytope $\mathbf{P}$ shows that $\lambda_{\text {sep }}(\mathbf{K})$ may be arbitrarily large for a three-dimensional smooth convex body $\mathbf{K}$. Indeed, if we take $\mathbf{K}:=\mathbf{P}+\varepsilon \mathbf{B}^{d}$ with a small $\varepsilon>0$, we obtain a smooth body for which, by the previous argument, $\lambda_{\text {sep }}(\mathbf{K})$ is large.

Thus, it would be very interesting to see a lower bound on $f(\mathbf{K})$ of Theorem 3 which depends on $d$ only.

\section{ACKNOWLEDGEMENTS}

Károly Bezdek was partially supported by a Natural Sciences and Engineering Research Council of Canada Discovery Grant. Márton Naszódi was partially supported by the National Research, Development and Innovation Office (NKFIH) grant NKFI-K119670 and by the János Bolyai Research Scholarship of the Hungarian Academy of Sciences, as well as the ÚNKP-17-4 New National Excellence Program of the Ministry of Human Capacities.

\section{REFERENCES}

[1] K. Ball, Volume ratios and a reverse isoperimetric inequality, J. London Math. Soc. (2) 44 (1991), no. 2, 351-359.

[2] K. Bezdek, On the maximum number of touching pairs in a finite packing of translates of a convex body, J. Combin. Theory Ser. A 98 (2002), no. 1, 192-200.

[3] K. Bezdek and P. Brass, On $k^{+}$-neighbour packings and one-sided Hadwiger configurations, Beiträge Algebra Geom. 44 (2003), no. 2, 493-498.

[4] K. Bezdek, M. Khan, and M. Oliwa, On contact graphs of totally separable domains, arXiv:1703.08568 [math] (2017).

[5] K. Bezdek and Zs. Lángi, Minimizing the mean projections of finite $\rho$-separable packings, Monatsh. Math. (published online) https://doi.org/10.1007/s00605-018-1166-y.

[6] K. Bezdek, B. Szalkai, and I. Szalkai, On contact numbers of totally separable unit sphere packings, Discrete Math. 339 (2016), no. 2, 668-676.

[7] P. Boyvalenkov, S. Dodunekov, and O. Musin, A survey on the kissing numbers, Serdica Math. J. 38 (2012), no. 4, 507-522.

[8] L. Danzer and B. Grünbaum, Über zwei Probleme bezüglich konvexer Körper von P. Erdős und von V. L. Klee, Math. Z. 79 (1962), 95-99.

[9] H. Davenport and Gy. Hajós, Problem 35, Mat. Lapok 2 (1951), 68.

[10] G. Fejes Tóth and L. Fejes Tóth, On totally separable domains, Acta Math. Acad. Sci. Hungar. 24 (1973), 229-232. 
[11] H. Groemer, Abschätzungen für die Anzahl der konvexen Körper, die einen konvexen Körper berühren, Monatsh. Math. 65 (1961), 74-81.

[12] H. Hadwiger, Über Treffanzahlen bei translationsgleichen Eikörpern, Arch. Math. 8 (1957), 212-213.

[13] G. Kertész, On totally separable packings of equal balls, Acta Math. Hungar. 51 (1988), no. 3-4, 363-364.

[14] W. Kuperberg, Optimal arrangements in packing congruent balls in a spherical container, Discrete Comput. Geom. 37 (2007), no. 2, 205-212.

[15] H. Minkowski, Dichteste gitterförmige Lagerung kongruenter Körper, Nachrichten von der Gesellschaft der Wissenschaften zu Göttingen, Mathematisch-Physikalische Klasse 1904 (1904), 311355.

[16] O. Musin, The kissing number in four dimensions, Ann. of Math. (2) 168 (2008), no. 1, 1-32.

[17] A. M. Odlyzko and N. J. A. Sloane, New bounds on the number of unit spheres that can touch a unit sphere in $n$ dimensions, J. Combin. Theory Ser. A 26 (1979), no. 2, 210-214.

[18] R. A. Rankin, The closest packing of spherical caps in $n$ dimensions, Proc. Glasgow Math. Assoc. 2 (1955), 139-144.

[19] J. R. Reay, Generalizations of a theorem of Carathéodory, Mem. Amer. Math. Soc. 54 (1965), 50.

[20] K. Schütte and B. L. van der Waerden, Das Problem der dreizehn Kugeln, Math. Ann. 125 (1953), $325-334$.

[21] E. Steinitz, Bedingt konvergente Reihen und konvexe Systeme, J. Reine Angew. Math. 143 (1913), $128-176$.

[22] I. Talata, Exponential lower bound for the translative kissing numbers of d-dimensional convex bodies, Discrete Comput. Geom. 19 (1998), no. 3, 447-455.

(K.B.) Department of Mathematics and Statistics, University of Calgary, Canada.

Department of Mathematics, University of Pannonia, Veszprém, Hungary.

E-mail address: bezdek@math.ucalgary.ca

(M.N.) Department of Geometry, Eötvös Loránd University, Budapest, Hungary

E-mail address: marton.naszodi@math.elte.hu 